\title{
Use of the distorted wave Born approximation to predict scattering by inhomogeneous objects: Application to squid
}

\author{
Benjamin A. Jones, a) Andone C. Lavery, and Timothy K. Stanton \\ Department of Applied Ocean Physics and Engineering, Woods Hole Oceanographic Institution, \\ Woods Hole, Massachusetts 02543-1053
}

(Received 8 August 2007; revised 7 August 2008; accepted 10 October 2008)

\begin{abstract}
A new method has been developed to predict acoustic scattering by weakly scattering objects with three-dimensional variability in sound speed and density. This variability can take the form of inhomogeneities within the body of the scatterer and/or geometries where the acoustic wave passes through part of the scattering body, into the surrounding medium, and back into the body. This method applies the distorted wave Born approximation (DWBA) using a numerical approach that rigorously accounts for the phase changes within a scattering volume. Ranges of validity with respect to material properties and numerical considerations are first explored through comparisons with modal-series-based predictions of scattering by fluid-filled spherical and cylindrical fluid shells. The method is then applied to squid and incorporates high resolution spiral computerized tomography (SCT) scans of the complex morphology of the organism. Target strength predictions based on the SCT scans are compared with published backscattering data from live, freely swimming and tethered squid. The new method shows significant improvement for both singleorientation and orientation-averaged scattering predictions over the DWBA-homogeneousprolate-spheroid model. (C) 2009 Acoustical Society of America. [DOI: 10.1121/1.3021298]
\end{abstract}

PACS number(s): 43.30.Ft, 43.30.Sf, 43.30.Zk [KGF]

Pages: $73-88$

\section{INTRODUCTION}

Extracting biologically important information such as organism type and abundance from acoustic scattering data relies heavily on the availability of experimentally validated scattering models [see reviews by Horne (2000) and Simmonds and MacLennan (2005)]. The application of a model often dictates the level of complexity used in representing the scatterer. For example, predictions of volume scattering, which may use an ensemble of discrete predictions averaged over a range of parameters such as size and orientation of the scatterer, do not need the level of complexity required to predict discrete echoes for unique identification of class, size, or species of the scattering organism.

Much research has focused on organisms, principally zooplankton, that are classified as weakly scattering with fluidlike material properties because of their high numerical abundance and importance in the ocean food web. Weakly scattering implies a density and sound speed similar to that of the surrounding water, whereas, fluidlike material properties refers to tissue that does not support shear waves. Significant advances have been made in developing acoustic scattering models for this group of organisms involving shapes of varying complexity. The representation of shape in early studies was based on models of simple geometric fluid volumes such as spheres, finite-length cylinders, and prolate spheroids (Anderson, 1950; Yeh, 1967; Johnson, 1977; Stanton, 1988). More recent models have incorporated two-

\footnotetext{
${ }^{a)}$ Present address: USS Bonhomme Richard (LHD 6), FPO AP 96617-1656. Electronic mail: bnyjones@gmail.com
}

dimensional (Stanton et al., 1988; McGehee et al., 1988; Amakasu and Furusawa, 2006; Stanton and Chu, 2000) and three-dimensional (Lavery et al., 2002) details of zooplankton shape.

One general approach that can account for anatomical complexities of weak scatterers is the distorted wave Born approximation (DWBA) (Chu et al., 1993; Stanton et al., 1993). This volume integral formulation for weakly scattering bodies can account for three-dimensional shape as well as inhomogeneous material properties. Accurately applying this formulation, however, requires digitizing an organism's complex inner and outer anatomy at sufficiently high resolution (Stanton and Chu, 2000), an issue addressed in this paper. The DWBA formulation has been successfully applied to model the scattering of sound from various types of zooplankton, including euphausiids, copepods, and decapod shrimp using high resolution outer shapes, although in these cases the changes in phase that occur when the acoustic wave passes through part of the scattering body into the surrounding medium, and back into the body were not taken into consideration. These model predictions have been validated through laboratory and, to some extent, field measurements (Simmonds and MacLennan, 2005; Lawson et al., 2006; Lavery et al., 2007; Stanton and Chu, 2000).

Accounting for internal anatomical structure in the DWBA and other models remains a challenge. One modeling restriction is the limited information available concerning sound speed and density of different tissues within most marine organisms. Even when these variations are known, implementation into the model can be a challenge. For both of these reasons, application of the DWBA has been prima- 
rily used to model marine organisms as homogeneous scatterers, as in most of the aforementioned studies. Application of the DWBA to inhomogeneous scatterers has been limited to a subset of those studies. In those exploratory cases the material properties were varied, somewhat heuristically, along the length of an organism's body, with the variations corresponding to segmentation of the exoskeleton (Stanton et al., 1988; Stanton and Chu, 2000; Lavery et al., 2002). Furthermore, those studies did not account for the influence of inhomogeneities on the phase of the incident acoustic wave.

Given the demonstrated usefulness of the DWBA when applied to homogeneous objects, the formulation is further explored in this paper to account for three-dimensional variations of material properties within the objects. Although the DWBA formulation does, in principle, account for threedimensional inhomogeneous material properties, evaluating the integral in this case requires careful consideration of the phase of the incident signal within the object. In the original Born approximation (undistorted), the amplitude and phase of the incident wave are only dependent on the position of the wavefront with respect to some arbitrary origin and the material properties of the surrounding medium. This is due to the general assumption that the incident wave is unmodified by the weakly scattering body. In contrast, the DWBA is a modification to the Born approximation in which the wavenumber inside the scattering volume is determined by the material properties within the body [e.g., see Stanton et al. (1993)].

The undistorted Born approximation has, in fact, been used to successfully describe scattering by inhomogeneous continuous random media, such as atmospheric and oceanic turbulence (Goodman, 1990; Lavery et al., 2003; Tatarski, 1961). However, when the Born approximation has been applied to finite bounded scatterers, such as spheres, cylinders, and zooplankton, it has been found that the frequency responses of the scattering, most notably the positions of nulls in these spectra, are not accurately predicted. It is this result, which originally motivated use of the DWBA (although not shown explicitly in the original papers), that more accurately predicts the position of the nulls in frequency spectra of the scatterers (Stanton et al., 1993; Chu et al., 1993). In the DWBA, the phase of the wavefront, at any point in the volume, is dependent both on the distance traveled by the incident wave and any sound speed variations encountered along the path traveled. Accounting for this dependence presents a significant challenge in applying the DWBA to inhomogeneous objects and is the subject of this paper.

The DWBA is applied to inhomogeneous bodies in this paper by accurately accounting for both the amplitude and phase change of the incident wave as it travels through a scattering volume. Sound speed and density can vary arbitrarily in three dimensions, and the only restriction is that the variations must be small compared with the average values of the material properties of both the scattering volume and the surrounding medium. The numerical implementation of this method uses a two-part algorithm that calculates phase and amplitude separately for every discretization before integrating over the entire volume. This method of solving the
DWBA is referred to as the phase-tracking DWBA method throughout this article. This approach to implementing the DWBA is first tested against analytical solutions to the simple cases of fluid-filled spherical and cylindrical fluid shells and then applied to the more complex case of squid.

Although relatively little work has been published on acoustic scattering models of squid, there is considerable interest in this research in two different areas. The first is commercial fisheries. Acoustic stock assessments of squid can be used to complement more traditional techniques such as trawl surveys by rapidly surveying large volumes of water and providing real time population assessments (Starr and Thorne, 1988). Understanding sound scattering by squid is essential for a quantitative interpretation of these acoustic surveys. Secondly, there is increasing interest in the predatorprey relationship between echolocating marine mammals and squid. Beaked whales, for instance, hunt squid using broadband ultrasonic sonar (Johnson et al., 2004; Madsen et al., 2005) and may use characteristics of the frequency responses from prey to help discriminate between targets in the water column (Jones et al., 2008). Scattering models that help define the dominant scattering mechanisms of squid may elucidate factors that are exploited by the whales in discriminating between prey and nonprey.

Measurements of density and sound speed of squid suggest that these invertebrates are well suited to being modeled as weak scatterers (Mukai et al., 2000; Kang et al., 2006; Iida et al., 2006). Accordingly, previous models of squid use the exact liquid prolate spheroid model and the DWBA formulation, also using a prolate spheroidal geometry (Arnaya and Sano, 1990; Mukai et al., 2000). However, these models assume homogeneous material properties within the scattering volume of simple shape. This assumption is clearly a simplification of the complex anatomy of squid. For example, the main section of the squid body includes the mantle, an outer shell of tissue that partially encloses a seawater-filled cavity called the mantle chamber.

In this study high resolution, spiral computerized tomography (SCT) scans of squid have been taken. The results of these scans enable the incorporation of the complex interior and exterior shape of the squid's body into the phasetracking DWBA method by differentiating between seawater-filled cavities and the squid's body. Appendages (i.e., arms, tentacles, and fin) as well as internal structure (i.e., the mantle cavity) are treated as inhomogeneities within the scattering volume. Material property variation due to internal organs, however, is not included in this study. Scattering predictions based on SCT scans of a locally caught squid are compared with published measurements of both anesthetized, tethered squid and live, freely swimming squid.

This paper is organized as follows: the phase-tracking DWBA for inhomogeneous objects is first developed. In order to assess the accuracy of this method, it is then applied to simple geometric shapes of both homogeneous and inhomogeneous material compositions for which there are analytical solutions. Two scattering formulations are used for comparisons. Modal-series-based scattering models (spherical shell and finite-length cylindrical shell) are used to compare predictions of scattering by objects with various-sized inhomo- 
geneities and a range of material properties. Since the modalseries-based solution for scattering from cylindrical shells applies only near-normal incidence, a DWBA-based finitelength cylinder model is used to compare predictions of scattering by homogeneous elongated bodies over all orientations. Finally, the phase-tracking DWBA method is applied to the more complex case of squid. Spiral CT scans provided high resolution three-dimensional measurements of a locally available species of squid, Loligo pealeii. These measurements were then scaled, and in one case altered, so that predictions of acoustic scattering could be compared with published scattering data involving squid of the species Todarodes pacificus. Conclusions are then made about the benefits of this new approach.

\section{THEORY}

\section{A. Definitions of fundamental quantities}

Acoustic scattering from an object in the far field can be described in terms of the amplitude of the incident sound wave, $P_{0}$, and the scattering amplitude, $f$,

$$
P_{\text {scat }}=P_{0} \frac{e^{\mathrm{i} k_{1} r}}{r} f
$$

where $r$ is the distance from the object to the receiver. The acoustic wavenumber of the surrounding medium, $k_{1}$, is defined as $2 \pi / \lambda_{1}$, where $\lambda_{1}$ is the acoustic wavelength in the surrounding medium.

Target strength (TS) is the logarithmic measure of the backscattered signal, expressed in decibels, relative to $\mathrm{m}^{2}$,

$$
\mathrm{TS}=10 \log \sigma_{\mathrm{bs}},
$$

where $\sigma_{\mathrm{bs}}=\left|f_{\mathrm{bs}}\right|^{2}$ is the differential backscattering cross section and $f_{\mathrm{bs}}$, or backscattering amplitude, is the scattering amplitude evaluated in the backscattering direction. Mean TS is determined by averaging $\sigma_{\mathrm{bs}}$ prior to logarithmic conversion and is given as $\langle\mathrm{TS}\rangle=10 \log \left\langle\sigma_{\mathrm{bs}}\right\rangle$.

In order to compare scattering from objects that are similar in proportion but of different overall sizes, the reduced target strength (RTS) is often used. Throughout this article scattering predictions will refer to either RTS or TS, which only differ by a constant. For a sphere or spherical shell of outer radius $a$ (Fig. 1), RTS is given by

$$
\mathrm{RTS}=10 \log \frac{\sigma_{\mathrm{bs}}}{\pi a^{2}} .
$$

In the case of elongated objects such as cylinders or cylindrical shells of length $L$, the RTS is given by

$$
\mathrm{RTS}=10 \log \frac{\sigma_{\mathrm{bs}}}{L^{2}}
$$

For the case of scattering from squid, the length $L$ is replaced by dorsal mantle length, $L_{\mathrm{ml}}$. Additionally, due to the lack of published data on mean mantle widths, from which mean cylindrical radii for squid could be derived, the nondimensional term $k_{1} L_{\mathrm{ml}}$ is used instead of the more commonly used $k_{1} a$ for scattering predictions.

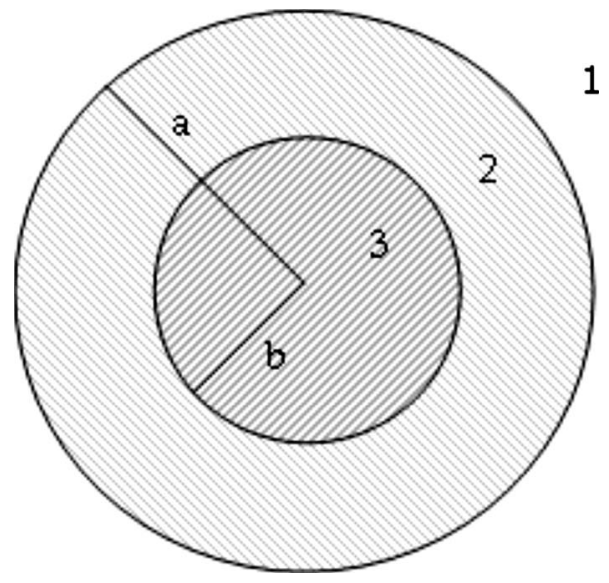

FIG. 1. Bisection of a spherical shell or cross section of a cylindrical shell. Indices 1-3 correspond to fluids of different material properties (i.e., sound speed, $c_{i}$, and density, $\left.\rho_{i}, i=1,2,3\right)$. The radii $a$ and $b$ are the outer and inner shell radii, respectively, such that $(a-b) / a$ corresponds to fractional shell thickness, $\tau$.

\section{B. The DWBA formulation}

The Born approximation was derived for weakly scattering bodies in which the total pressure field inside a scattering volume is approximated by the unperturbed incident field. The approximation is valid when the amplitude of the scattered wave is much smaller than the incident wave (Morse and Ingard, 1968). Due to its volume integral form, this approximation may be used for arbitrarily shaped scatterers with any orientation with respect to the incident sound wave. Furthermore, it is valid for all acoustic frequencies. The DWBA formulation involves replacing the incident wavenumber vector inside the integral with the wavenumber vector resulting from the local sound speed and the frequency of the incident sound wave. It is this modification that accounts for the phase change of the sound wave due to material property inhomogeneities within the scattering volume. Within this formulation, Eq. (8) of Stanton et al. (1993), the backscattering amplitude is given by

$$
f_{\mathrm{bs}}=\frac{k_{1}^{2}}{4 \pi} \iiint_{v}\left(\gamma_{\kappa}-\gamma_{\rho}\right) e^{2 \mathrm{i} k_{v} \cdot r_{v}} d v \text {. }
$$

The terms $\gamma_{\kappa}$ and $\gamma_{\rho}$ are defined in terms of compressibility, $\kappa$, and density, $\rho$, or can also be expressed in terms of the commonly used ratios of density, $g_{v} \equiv \rho_{v} / \rho_{1}$, and sound speed, $h_{v} \equiv c_{v} / c_{1}$, given here by

$$
\begin{gathered}
\gamma_{\kappa} \equiv \frac{\kappa_{v}-\kappa_{1}}{\kappa_{1}}=\frac{1-g_{v} h_{v}^{2}}{g_{v} h_{v}^{2}}, \\
\gamma_{\rho} \equiv \frac{\rho_{v}-\rho_{1}}{\rho_{v}}=\frac{g_{v}-1}{g_{v}} .
\end{gathered}
$$

In all cases the subscript " 1 " indicates parameters of the surrounding medium and the subscript " $v$ " indicates parameters within the scattering volume. Accordingly, the wavenumber in the external medium is the constant $k_{1}$, while the wavenumber vector and the position vector within the volume are $\boldsymbol{k}_{v}$ and $\boldsymbol{r}_{v}$, respectively. Throughout this paper, when a model formulation is applied to a scattering problem, the 
subscript " $v$ " takes on the integers " $2,3, \ldots$ " corresponding to given material property parameters of that region within the volume. For example, in the region designated by " 3 " in Fig. 1 , the density ratio, $g_{v}$, becomes $g_{3}=\rho_{3} / \rho_{1}$.

In Stanton et al. (1998), this formulation was simplified to a line integral for objects that are axisymmetric at any point along their lengthwise (deformed) axis. For straight, finite-length cylinders, the line integral can be further reduced to the following closed form equation:

$$
\begin{aligned}
f_{\mathrm{bs}}= & \frac{k_{1} k_{v} a^{2} L}{2}\left(\gamma_{\kappa}-\gamma_{\rho}\right) e^{-\mathrm{i} L k_{v} \sin \theta} \\
& \times \frac{J_{1}\left(2 k_{v} a \cos \theta\right)}{2 k_{v} a \cos \theta} \frac{\sin \left(k_{v} L \sin \theta\right)}{k_{v} L \sin \theta},
\end{aligned}
$$

where $J_{1}$ is the Bessel function of the first kind and $\theta$ is the angle of incidence measured from the incident wave vector, $\boldsymbol{k}_{1}$, to a plane orthogonal to the longitudinal axis of the cylinder (e.g., $\theta=0$ is normal incidence). It should be noted that the exponential phase term is dependent on the zero-phase origin used in the calculation, although this does not affect the calculations of TS or RTS, which use the absolute value of $f_{\mathrm{bs}}$. This model is termed the DWBA finite-length cylinder model (closed form) throughout this paper and is valid over all angles of orientation. This result is used to test the phasetracking DWBA approach over a range of orientations of the scattering volume with respect to the incident acoustic wave.

\section{Numerical implementation of the phase-tracking DWBA method}

The DWBA volume integral formulation can, in principle, account for cases where the scattering body has inhomogeneous material properties or where the wave passes through part of the body, into the surrounding medium, and back into the body. However, evaluating the integral for these conditions is a challenge as the phase must be calculated at each point in the volume by piecewise integration of the exponential phase term. In other words, the phase at any given point depends not only on the local sound speed but also on the variation in sound speed over the path the sound wave has already traveled through the scattering body. Given the complexity of the problem, a numerical approach has been developed to implement the DWBA for inhomogeneous objects.

The model presented in this article, which numerically computes the DWBA volume integral, is based on discretizing the scattering volume. The volume is composed of $l$ cross-sectional matrices, $M^{l}$. Each element of each matrix, $M_{i j}^{l}$, represents one volume element, or voxel, and is assigned an integer value depending on the material properties of that voxel. Although only two fluid types are used to represent scattering volumes in this study, this model can, in general, be applied to volumes containing any arbitrary variation of material properties. Both internal inhomogeneities and geometries where the sound wave has multiple points of entry into and exit from the scattering body are treated as inhomogeneous media (see, for example, Fig. 2).

In order to accurately account for the phase of the sound wave as it propagates through an inhomogeneous medium, it
Multiple entrances and exits

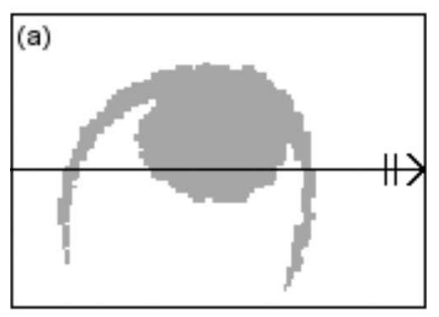

Internal inhomogeneities
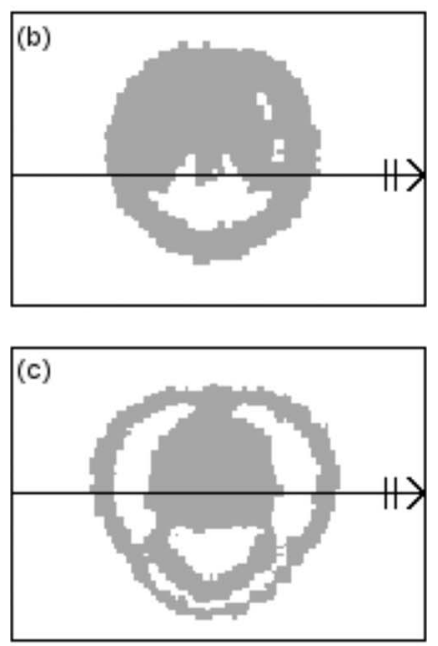

Homogeneous

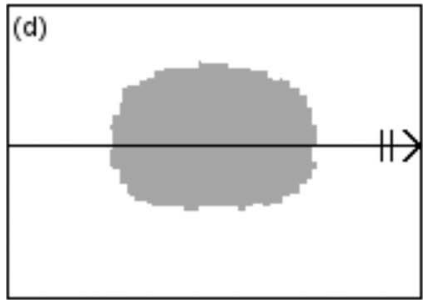

FIG. 2. Ray diagrams, using actual SCT scans of L. pealeii, that illustrate various types of inhomogeneous and homogeneous scattering volumes including (a) squid body and fin: an irregularly shaped object in which the ray path is traced alternately into squid tissue and seawater (treated as an inhomogeneous medium), [(b) and (c)] squid mantle and mantle cavity: a continuous external boundary with internal inhomogeneities, and (d) squid neck: a homogeneous volume.

is necessary to incorporate a phase-tracking algorithm. This algorithm, analogous to a ray-tracing routine, calculates the phase change associated with the sound speed of each differential element along a path traversed by the sound wave. For backscattering, the one-way phase change is simply doubled to calculate the full phase term.

The cumulative phase change is calculated by first digitally rotating the volume matrix using "nearest neighbor" interpolation, such that the incident wavenumber vector, $\boldsymbol{k}_{1}$, is orthogonal to the new cross sections $\tilde{M}^{l}$. The rotation limits the phase-tracking to a one-dimensional problem. The differential phase change is calculated for each voxel from the locally assigned sound speed and differential length over which the sound wave travels. The total one-way phase change is then found as the cumulative summation of these differential phase changes from the zero-phase reference plane to this voxel. 
Finally, a search routine creates a linear index of all voxels within the rotated volume assigned a particular material property and matches the appropriate amplitude term with the round-trip-phase term. Using a Cartesian coordinate system $(x, y, z)$, the analytical expression given in Eq. (5) becomes

$$
f_{\mathrm{bs}}=\frac{k_{1}^{2}}{4 \pi} \sum_{l=1}^{N_{z}} \sum_{j=1}^{N_{y}} \sum_{i=1}^{N_{x}}\left(\gamma_{\kappa}-\gamma_{\rho}\right)_{i j}^{l} e^{2 \mathrm{i} \varphi_{i j}^{l}} d v,
$$

where $d v$ is the product of the differential lengths, $d x, d y$, and $d z$, and the phase term, $\varphi_{i j}^{l}$, is a cumulative summation of the phase change, in the $z$ direction, and is given by

$$
\varphi_{i j}^{l}=\sum_{q=1}^{l} k_{i j}^{q} d z .
$$

\section{Modal-series-based scattering models}

In order to validate the phase-tracking DWBA method for inhomogeneous objects, comparisons are made with analytical modal-series-based solutions for scattering from simple geometric, shelled, and hence inhomogeneous objects. The wave equation can be separated and solved exactly for a limited number of simple shapes (e.g., spheres, infinite cylinders, and prolate spheroids), resulting in modal series solutions. The modal series solutions for these simple geometric volumes are derived, using the far-field condition, after separating the wave equation in the respective coordinate system and applying two boundary conditions at each interface: continuity of pressure and continuity of radial velocity. In this section scattering models are presented for fluid-filled spherical and cylindrical (finite-length) fluid shells that are based on these modal series solutions.

The solutions to the spherical and infinite cylindrical shells are exact. The model for the finite-length cylindrical shell, however, is adapted from the latter case and is an approximation. This approximation neglects end effects and is, therefore, accurate only for near-normal incidence. Accordingly, this formulation is only applied at normal incidence to test the phase-tracking DWBA model over a range of frequencies, shell thicknesses, and material properties.

\section{Fluid-filled spherical fluid shell}

The far-field modal-series-based solution to the wave equation for a fluid-filled spherical fluid shell in the backscattered direction is expressed as

$$
f_{\mathrm{bs}}=\frac{\mathrm{i}}{k_{1}} \sum_{n=0}^{\infty}(2 n+1)(-1)^{n} A_{n}
$$

where $A_{n}$ is the $n$th modal series coefficient and is given in the Appendix. This solution is valid for all sound speeds and densities, shell thicknesses, and acoustic frequencies.

\section{Fluid-filled cylindrical (finite-length) fluid shell}

Stanton (1988) related the scattering amplitude of a finite-length cylinder to that of an infinite-length cylinder using an approximation in which the volume flow per unit
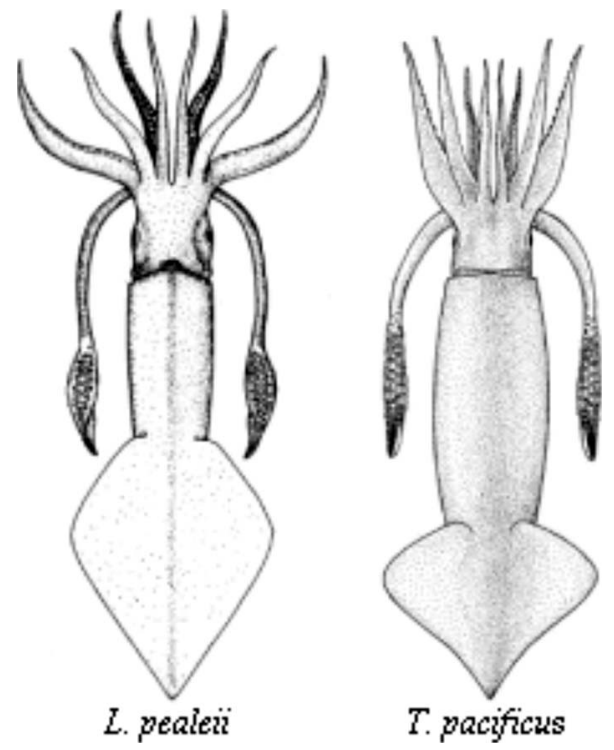

FIG. 3. Sketches of the species of squid discussed in this article (Roper et al., 1984) The L. pealeii, shown on the left, is the littoral species found near Woods Hole, MA, from which high resolution morphometry was obtained using SCT scans. The T. pacificus shown on the right, a pelagic species found in the western Pacific Ocean, has a smaller aspect ratio (length-to-width ratio). Published acoustic scattering data from experiments involving $T$. pacificus are used to compare with phase-tracking DWBA predictions.

length of the scattered field of the cylinder is approximated by that of the infinite cylinder. These equations can, likewise, be applied to a finite cylindrical shell. Using the same approximation, the backscattering by a fluid-filled cylindrical (finite-length) fluid shell at normal incidence is

$$
f_{\mathrm{bs}}=\frac{\mathrm{i} L}{\pi} \sum_{n=0}^{\infty}-\epsilon_{n}(-1)^{n} B_{n},
$$

where $B_{n}$ is the $n$th modal series coefficient for the infinitelength fluid-filled cylindrical fluid shell and is given in the Appendix. The term $\epsilon_{n}$ is the Neumann number $\left(\epsilon_{0}=1, \epsilon_{n}\right.$ $=2$ for $n=1,2,3, \ldots)$.

\section{MATERIALS AND METHODS}

\section{A. Animals studied}

Two species of squid are used in this research (Fig. 3). The morphometry of a locally available long-finned squid, L. pealeii, is measured at high resolution, while published target strength data are available for a second species, $T$. pacificus, or the Japanese common squid. Scattering predictions are made based on the three-dimensional morphology of $L$. pealeii, digitally scaled to match the aspect ratio (i.e., length-to-width ratio) of a $T$. pacificus and compared with published data. In one case, partial scans of two different $L$. pealeii were combined so that the arms and tentacles of the free-swimming T. pacificus could best be modeled.

\section{Long-finned squid, Loligo pealeii}

L. pealeii specimens, obtained from the Marine Biological Laboratory, Woods Hole, MA, were selected due to ease of availability and well documented details of their anatomy. 
TABLE I. Material properties of squid from published sources. Values given as a ratio of squid material properties to those of seawater in the same publication.

\begin{tabular}{cccc}
\hline \hline Reference & Material & $g$ & $h$ \\
\hline Hashimoto and Maniwa (1952) & Whole squid & $\ldots$ & $1.007^{\mathrm{b}}$ \\
Mukai et al. (2000) & Whole squid & 1.025 & $\ldots$ \\
Kang et al. $(2006)$ & Whole squid & 1.029 & 1.041 \\
Iida et al. (2006) & Mantle tissue & 1.043 & 1.053 \\
\hline \hline
\end{tabular}

${ }^{\mathrm{a} I n}$ Japanese, cited by Kang et al. (2006).

${ }^{\mathrm{b}}$ Based on assumed sound speed in seawater of $1500 \mathrm{~m} / \mathrm{s}$.

This coastal species of squid has a long, slender body and a large fin in proportion to its mantle length. These squid reach maturity at a mantle length of approximately $13-15 \mathrm{~cm}$ and approach a maximum mantle length of $40-50 \mathrm{~cm}$ (Roper et al., 1984).

\section{Japanese common squid, Todarodes pacificus}

T. pacificus is an oceanic squid with a muscular, moderately slender body and a relatively short fin. Members of this species reach maturity at a mantle length of $17-25 \mathrm{~cm}$ and a maximum size of $50 \mathrm{~cm}$ (Roper et al., 1984). Although these squid have a smaller aspect ratio than $L$. pealeii, the two species are broadly similar (Fig. 3).

\section{Squid material properties}

Acoustic scattering predictions require accurate values of sound speed and density of the scatterer. This is especially true of weakly scattering organisms whose material properties may vary from the surrounding water by only several percent (Chu et al., 2000). Very little has been published on squid material properties (Table I). However, some recent measurements provide useful information for this study. For example, Kang et al. (2006) measured average density and sound speed of whole anesthetized squid in seawater. Their estimates of sound speed as compared to previously reported values show better agreement with measured TS when used as parameters in a Kirchhoff ray mode model. Iida et al. (2006) recently measured values of sound speed and density of squid mantle tissue. These values are higher than those reported for whole squid. This is expected, however, as these measurements are for a single tissue type, whereas the whole squid measurements are average values of sound speed and density through both the squid tissue and the seawater-filled mantle cavity. In light of these results, the values given by Iida et al. (2006) were used in the phase-tracking DWBA method $\left(g_{v}=1.043\right.$ and $\left.h_{v}=1.053\right)$ to represent the various parts of the squid's body (i.e., mantle, internal organs, etc.). Seawater-filled cavities were given values of 1 for $g_{v}$ and $h_{v}$.

\section{B. High resolution morphometry of squid: SCT scans}

SCT scans were taken of a live, anesthetized squid (Fig. 4) and of a dead, previously frozen squid at the Marine Research Facility at the Woods Hole Oceanographic Institution. A spiral CT instrument conducts measurements in a helical pattern by taking a circular scan while the body is continu-

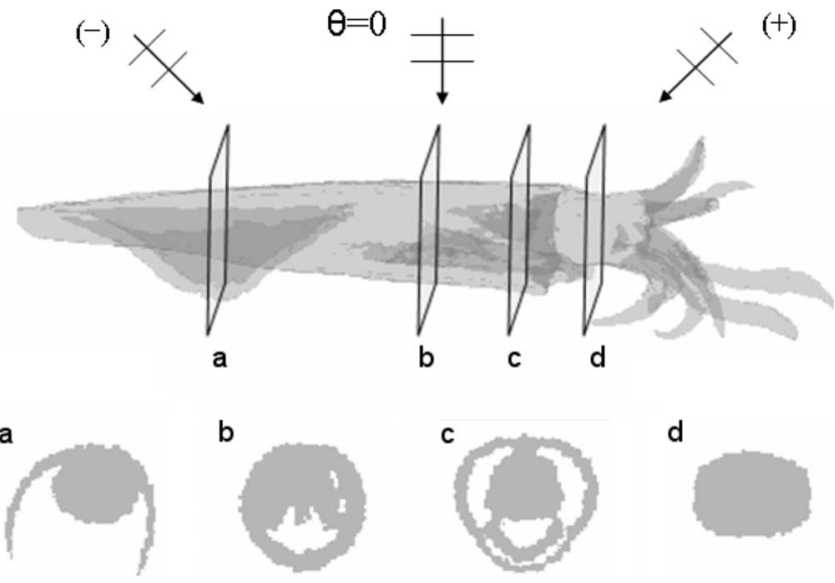

FIG. 4. Volume rendering composed of SCT scan images of L. pealeii (in semitransparent gray-scale) and four binary cross sections, $M_{l}$, showing the (a) fin, [(b) and (c)] mantle cavity, and (d) neck. In the cross sections, gray indicates squid body and white indicates surrounding seawater and seawater-filled cavities. Arrows show the orientation of the squid with respect to an incident sound wave $(-/+$ values of $\theta$ correspond to the head being down/up, respectively, and $\theta=0$ corresponds to normal incidence).

ously advanced through the scanning aperture. Cross sections are then reconstructed by interpolation into twodimensional images, with each pixel containing the x-ray attenuation in Hounsfield units (Hofer, 2000).

The live squid measured in this study was anesthetized in a $1 \%$ ethanol seawater solution. During scanning, the animal was suspended vertically in seawater to minimize distortion of body shape caused by laying the animal, unsupported, on a hard surface. A contrast agent was added to the seawater to help distinguish between $x$-ray attenuation of the solution and the squid body. The animal did not recover from the anesthesia and died either during the scanning process or soon after completion of the scan. The second specimen, a previously frozen dead squid was measured while suspended vertically in air. Scans of both specimens were taken by a Siemens Volume Zoom four-slice SCT scanner using pitch and collimator settings of 2 and $1 \mathrm{~mm}$, respectively. Images of $512 \times 512$ pixels were reconstructed from the data using $0.5 \mathrm{~mm}$ slice thickness. Individual pixel size was 0.5078 $\times 0.5078 \mathrm{~mm}^{2}$. Images were later resized on separate mathematical software using bicubic interpolation such that all pixels represent one cubic volume element, or voxel, with dimensions, $l_{\mathrm{vs}}$, equal to $0.5 \times 0.5 \times 0.5 \mathrm{~mm}^{3}$. In this way dimensional ratios are preserved in the image rotations discussed in the next section.

\section{Numerical implementation of SCT scans}

The SCT images of squid were cropped to the size of the largest cross section and then converted to binary matrices, $M^{l}$, using a simple threshold technique (Fig. 4). Scattering predictions were made by applying Eq. (9) to the resultant three-dimensional matrix. For incorporation into the phasetracking DWBA method (inhomogeneous case), each voxel, $M_{i j}^{l}$, identified as seawater, both inside and outside the body, was assigned a value of 0 , while voxels representing the squid's body were assigned a value of 1 . Tissue sound speed and density could not be directly inferred from SCT data. Thus, for modeling purposes all tissues not identified as sea- 
water were assumed to have material properties of mantle tissue. The binary conversion was insensitive to the threshold level in the cases of both squid scanned for this study. In the live, anesthetized case the contrasting agent made the seawater significantly higher in x-ray attenuation than the squid's body. In the case of the dead specimen scanned in air, the reverse was true, with the squid's body exhibiting much higher x-ray attenuation than the surrounding air.

\section{Scaled volume}

In order to model scattering by $T$. pacificus, the morphometry derived from the SCT scans of $L$. pealeii was scaled so that the aspect ratio (i.e., the ratio of mantle length to mantle width) was approximately equal to that of the $T$. pacificus. The aspect ratio of T. pacificus is approximately $10 \%$ smaller than that of L. pealeii.

\section{Hybridized volume}

One focus of this study was to incorporate a realistic swimming position of squid by using SCT scans of the live, anesthetized specimen. However, the anesthetized squid splayed its arms outward (Fig. 4) in a position that is not representative of the squid's natural swimming position (i.e., arms and tentacles folded into an anvil shape). In order to obtain a more realistic swimming morphometry, SCT scans of the dead squid, of similar size to the anesthetized squid, were taken with arms and tentacles folded together. The measurements of the more naturally positioned arms and tentacles from this animal were used to create a hybrid squid with the anesthetized squid's body. This hybrid squid was scaled as discussed in the previous section and used in the phase-tracking DWBA model. These predictions are compared with phase-tracking DWBA predictions for the nonhybridized live squid and with published measurements.

\section{Numerical issues related to phase-tracking approach}

The accuracy of digitization of a volume limits the range of frequencies for which the phase-tracking DWBA method can be applied. In general the ratio of acoustic wavelength to maximum voxel dimension should be about 20:1 although this requirement depends on the modeling approach used (Stanton and Chu, 2000). A closely related limitation is the computational time. Calculating the frequency-dependent backscattering amplitude, $f_{\mathrm{bs}}$, for broadband sound, where many finely incremented discrete frequencies are considered, can take a significant amount of computing time. Similarly, calculating the scattering as a function of orientation of the object at a discrete frequency is computationally intensive. In this model calculating $f_{\mathrm{bs}}$ as a function of orientation required significantly more processing time than calculating $f_{\mathrm{bs}}$ as a function of frequency, per discretization, due to the digital rotation of the volume.

\section{RESULTS}

\section{A. Scattering from fluid-filled spherical and cylindrical (finite-length) fluid shells}

The phase-tracking DWBA method is applied to computer-generated volumes of two simple shapes, spherical and finite-length cylindrical shells. In both cases the shell itself and interior volume are fluid materials. Phase-tracking DWBA predictions for spherical shells are compared with the exact modal series solution for fluid-filled spherical fluid shells [Eq. (11)]. Scattering predictions of cylindrical shells are compared, at normal incidence, with the modal-seriesbased solution for fluid-filled cylindrical (finite-length) fluid shells [Eq. (12)] and at various orientations for homogeneous finite-length cylinders with the DWBA finite-length cylinder model (closed form) [Eq. (8)].

\section{Shell thickness}

Scattering predictions for fluid shells of various thicknesses are compared with modal-series-based results to verify that the phase-tracking DWBA method accurately accounts for inhomogeneities (Figs. 5 and 6). Shell thickness is used as a proxy for various-sized inhomogeneities. Cylindrical and spherical shells, with fractional shell thickness, $\tau$ (see definition in Fig. 1 caption) varying from $100 \%$ (i.e., homogeneous) to $10 \%$, are used for comparison. In all cases, the density and sound speed of the shells are set to $1 \%$ greater than both the interior fluid and the surrounding fluid. All predictions show that scattering from spherical shells and cylindrical shells at normal incidence is strongly affected by the presence of inhomogeneities. Small scale structure was observed due to interference between waves scattered from the front and back interfaces of the object. While these interference patterns, for differing shell thicknesses, are nearly identical in terms of null spacing, the large scale structure varies significantly.

Comparison between the phase-tracking DWBA predictions and the modal series solutions shows excellent agreement. In the spherical case (Fig. 5), the numerical model predictions were generally within $\pm 1 \mathrm{~dB}$ of the modal series solution for all values of shell thicknesses up to values of $k_{1} a$ of $12.5\left(\lambda / l_{\mathrm{vs}}=50.3\right)$ and within $\pm 2 \mathrm{~dB}$ up to values of $k_{1} a$ of $17.5 \mathrm{~s}\left(\lambda / l_{\mathrm{vs}}=35.9\right)$. In the cylindrical case at normal incidence (Fig. 6) the phase-tracking DWBA predictions matched the modal-series-based solution generally within $\pm 0.5 \mathrm{~dB}$ for $k_{1} a$ up to $5\left(\lambda / l_{\mathrm{vs}}=50.3\right)$ and $\pm 2 \mathrm{~dB}$ for $k_{1} a$ $\leqslant 12.5\left(\lambda / l_{\mathrm{vs}}=20.2\right)$. In both cases some large discrepancies $(>10 \mathrm{~dB})$ existed; however, these were isolated to nulls of very low scattering values. As discussed in a following section, the disagreement between the phase-tracking DWBA predictions and modal-series-based solutions at higher values of $k_{1} a$ is a numerical issue related to voxel resolution. Further comparisons using homogeneous spheres of increased resolution confirm that the disagreement seen at higher $k_{1} a$ is primarily due to resolution issues (results not shown).

\section{Material properties}

As discussed above, the DWBA formulation is only valid for weak scatterers. In order to investigate the range of 


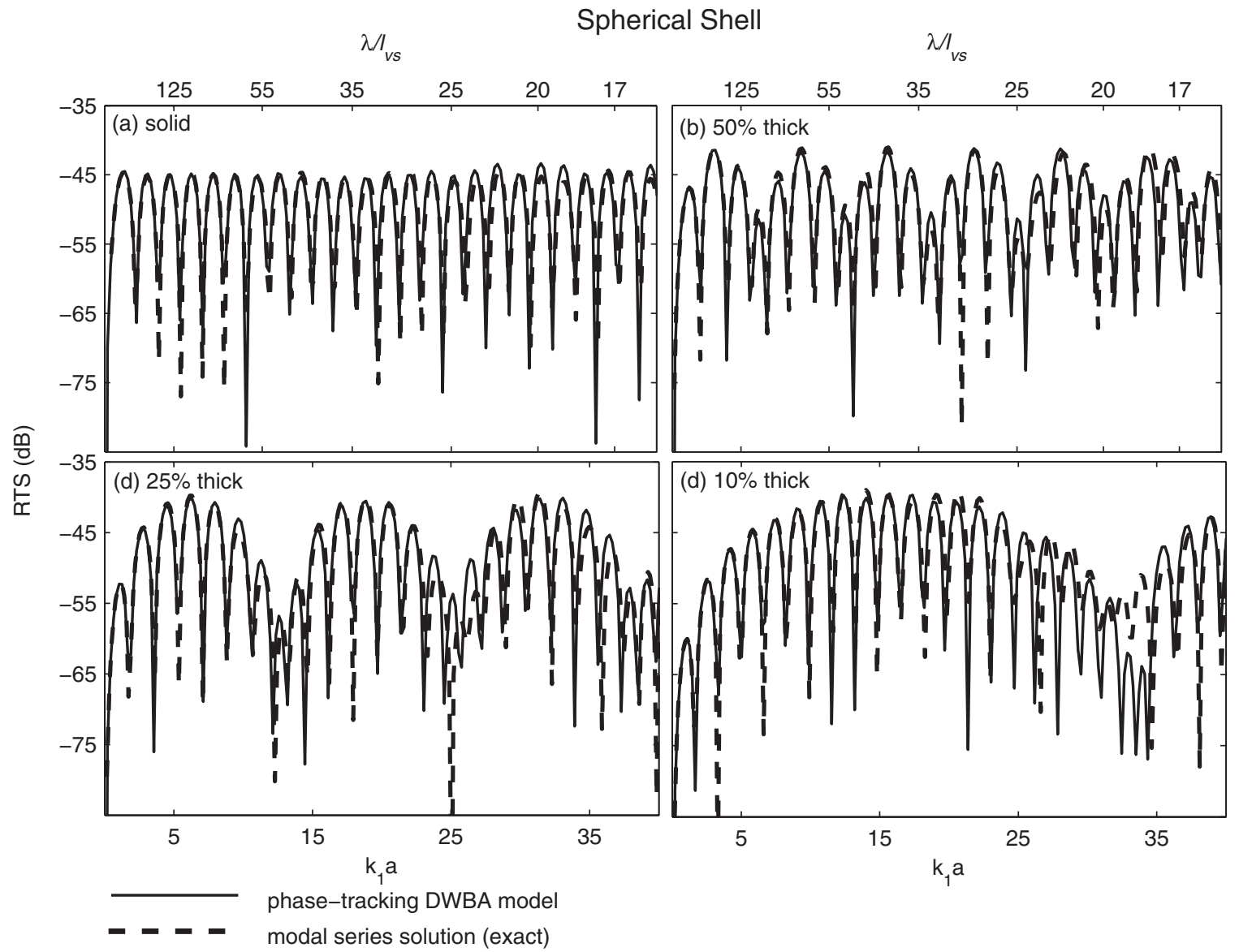

FIG. 5. Effect of varying shell thickness on backscattering by a fluid-filled spherical fluid shell. Phase-tracking DWBA predictions (solid lines) are compared with exact modal series solutions [Eq. (11)] (dashed lines). [Parameters: material properties: $g_{2}=1.01, h_{2}=1.01, g_{1}=g_{3}=1, h_{1}=h_{3}=1$, and $l_{\mathrm{vs}}=0.01 a$ ]

accuracy for the phase-tracking DWBA method with respect to material properties, finite-length cylindrical shells with various sound speed and density contrasts are considered. Model predictions at normal incidence are compared with the modal-series-based fluid-filled cylindrical (finite-length) fluid shell solution [Eq. (12)] that can describe a wide range of material profiles.

Scattering predictions were made using this model for finite-length cylindrical shells with fractional shell thickness of $25 \%$ and $g$ and $h$ values ranging from 1.02 to 1.06 (results not shown). Frequency was varied such that the maximum $k_{1} a$ ranged from 0 to 10 , which equates to a minimum resolution of $\lambda / l_{\mathrm{vs}} \simeq 25$. As a result of increasing sound speed and density contrast, the values of RTS increased correspondingly. There was excellent agreement outside the deepest portion of the nulls $( \pm 1 \mathrm{~dB})$ for $k_{1} a \leqslant 5\left(\lambda / l_{\mathrm{vs}} \simeq 50\right)$. Errors of up to $2 \mathrm{~dB}$ were seen at higher values of $k_{1} a$ and are largely due to resolution issues discussed in a following section.

\section{Orientation of scattering object}

In order to test the phase-tracking DWBA method for sound waves incident on an object at various angles, scattering from a homogeneous finite-length cylinder at a fixed value of $k_{1} a$ and varying tilt angles, $\theta$, was considered. Since the modal-series-based solution for finite cylinders is only accurate near broadside incidence, the phase-tracking
DWBA method was compared with the DWBA finite-length cylinder model (closed form) [Eq. (8)], which is valid for all orientations. When evaluated at the highest resolution, the phase-tracking DWBA method showed very good agreement with the analytic solution $( \pm 1 \mathrm{~dB})$ except in the deepest nulls [Fig. 7(a)].

It should be noted that the value chosen for $k_{1} a$ (5.03) in this portion of the study was neither in the deepest part of the null nor at a peak in the frequency response curve [Fig. 6(a)]. As seen in all the modeling results [Figs. 5-7 and 7(a)], the accuracy of the phase-tracking DWBA predictions at very low scattering values (i.e., near or in very deep nulls) is worst. This discrepancy is magnified because of the logarithmic scale. It is expected that this error for low values of predicted scattering is somewhat mitigated by the fact that very deep nulls are not as common in real organisms as in ideal shapes. The presence of these nulls in scattering from ideal shapes comes from nearly complete destructive interference of coherent scattering from the front and back interfaces.

\section{Effects of varying voxel resolution}

In order to test the accuracy of the phase-tracking DWBA method at different voxel resolutions, scattering predictions for homogeneous, cylindrical volumes of the same dimensions and varying voxel size, $l_{\mathrm{vs}}$, are compared to the DWBA finite-length cylinder model (closed form) [Eq. (8)]. 


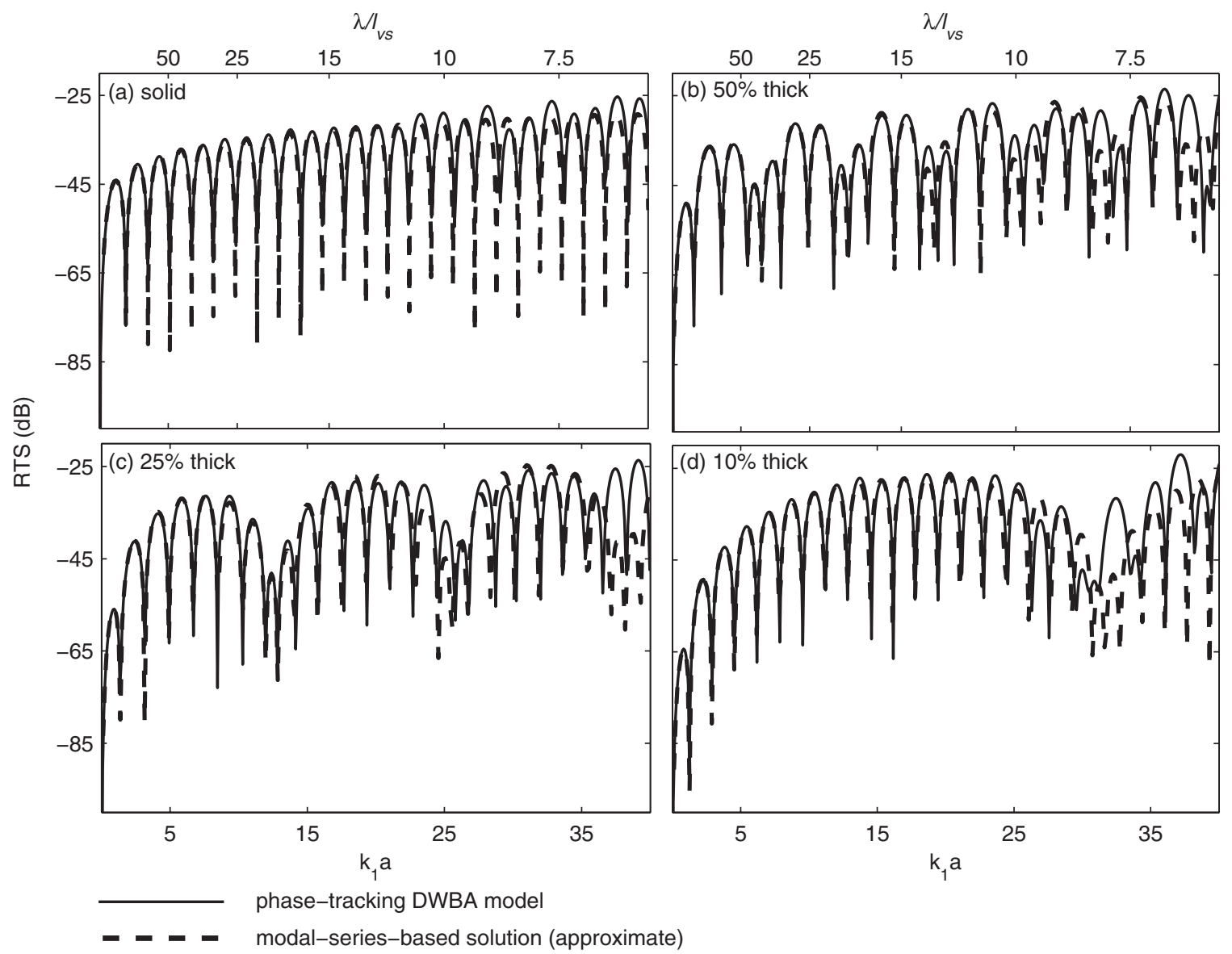

FIG. 6. Effect of varying shell thickness on backscattering by a fluid-filled cylindrical (finite-length) fluid shell. Phase-tracking DWBA predictions (solid lines) are compared with an approximate modal-series-based solution [Eq. (12)] (dashed lines). [Parameters: aspect ratio: $L / 2 a=2.5$; angle of incident wave: $\theta=0$ (normal incidence); material properties: $g_{2}=1.01, h_{2}=1.01, g_{1}=g_{3}=1, h_{1}=h_{3}=1$, and $l_{\mathrm{vs}}=0.025 a$. $]$

Resolution, expressed as wavelength with respect to voxel size $\left(\lambda / l_{\mathrm{vs}}\right)$, is varied from 75 to 12.5 for the RTS versus orientation predictions given in Figs. 7(a)-7(d). The model predictions matched the analytical solution fairly well for the large scale structure at a minimum resolution of 12.5 and were considerably better at a resolution of 25 . The small scale structure was generally within $\pm 2 \mathrm{~dB}$ at a resolution of 50.

The effects of resolution can also be seen in the results of broadside scattering from simple geometrical shapes. Figures 5 and 6 show how increasing $k_{1} a$, thus decreasing $\lambda / l_{\mathrm{vs}}$, affects the predictions. In the case of a spherical shell (Fig. 5 ), an accuracy of $\pm 2 \mathrm{~dB}$ requires a resolution of approximately 35 . In the cylinder case a similar accuracy can be obtained by a resolution of approximately 20 .

Although no clear pattern emerged, it has been shown that the resolution at which an object needs to be digitized for this phase-tracking DWBA approach depends on the scattering geometry. For an object with two-dimensional curvature, as with a cylinder, a minimum resolution of 20 provides an accuracy at normal incidence of $\pm 2 \mathrm{~dB}$, whereas an object with three-dimensional curvature, as in the case of a sphere, requires a somewhat higher level of resolution $\left(\lambda / l_{\mathrm{vs}} \geqslant 35\right)$. Finally, predictions of scattering by a cylinder over all angles of incidence require a significantly higher resolution for similar accuracy $\left(\lambda / l_{\mathrm{vs}} \geqslant 50\right)$.

\section{B. Application to squid}

The phase-tracking DWBA method with high resolution morphology derived from SCT scans of squid is first used to compare homogeneous and inhomogeneous model predictions for squid. Then, inhomogeneous model predictions are compared with measurements of scattering by live, freely swimming squid (Arnaya et al., 1989b, 1989c; Kang et al., 2005), as well as by tethered, anesthetized squid (Kang et al., 2005). These comparisons are made using both singleorientation and tilt-averaged results, where tilt is defined as rotation about the lateral axis of the squid (e.g., positive tilt is head up). All measurements of scattering by live squid concern only the species T. pacificus. Material properties for squid tissue and swimming tilt-angle distributions come from published sources, and, therefore, there were no floating parameters used in these predictions.

\section{Effect of inhomogeneities on scattering predictions}

In order to analyze the effects of including internal inhomogeneities (i.e., the seawater-filled mantle cavity) in modeling predictions, phase-tracking DWBA predictions using the high resolution morphometry of the live, anesthetized squid are compared for the homogeneous and inhomogeneous cases. Scattering predictions were made versus $k_{1} L_{m l}$ for dorsal aspect backscattering at various angles of tilt. The 

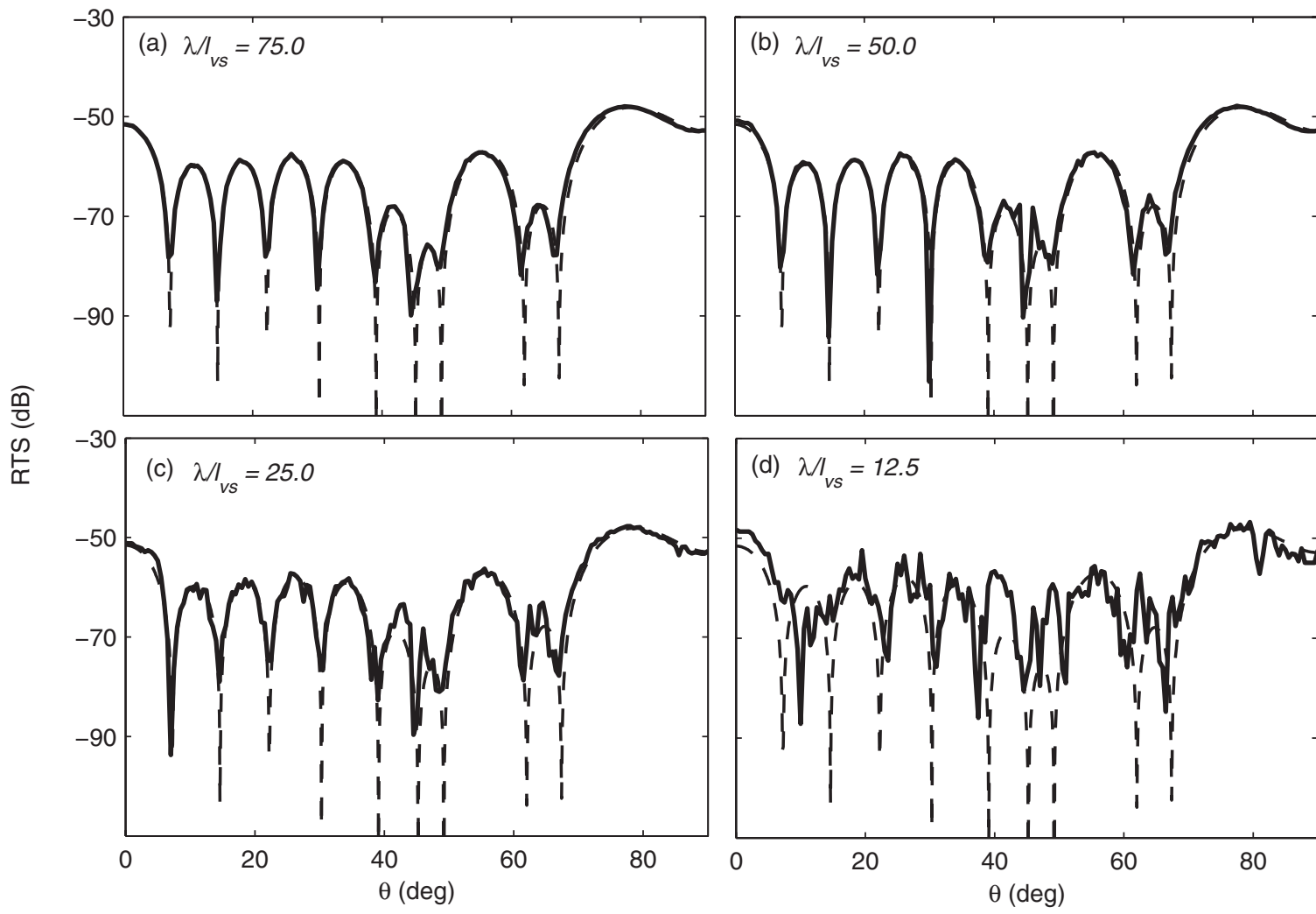

(d) $\lambda / I_{v s}=12.5$
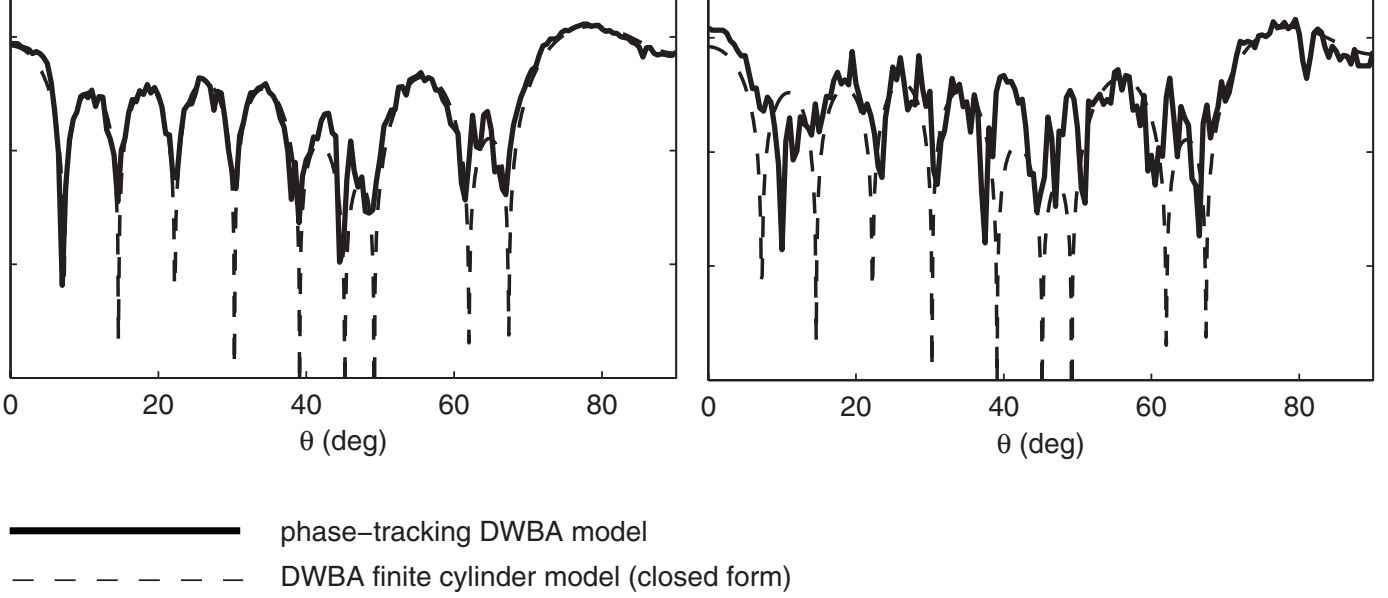

-

FIG. 7. Predictions of backscattering vs orientation for a homogeneous finite-length cylinder. Phase-tracking DWBA predictions (solid lines) are compared with DWBA finite-length cylinder model (closed form) [Eq. (8)] (dashed lines). [Parameters: $g=1.01, h=1.01, k_{1} a=5.03$, and aspect ratio, $L / 2 a=2.5$.]

mantle chamber represents $5.6 \%$ of the squid's body by volume. The effect of including this seawater-filled cavity is generally a $\pm 1-3 \mathrm{~dB}$ difference outside the deepest nulls as illustrated by predictions at four discrete angles ranging from $-30^{\circ}$ to $+30^{\circ}$ [Figs. 8(a)-8(d)], with the inhomogeneous model tending to predict higher TSs.

The effect of varying the position of the appendages, also treated by the phase-tracking DWBA method as volume inhomogeneities, can be seen by comparing the model predictions for the splayed-arms case and the straight-arms, hybrid-construction case with published measurements at various tilt angles (Fig. 9). By incorporating the more natural swimming position of the squid arms and tentacles (straightarms case), scattering predictions were improved for a range of angles off-normal incidence $\left(-20^{\circ}<\theta<-10^{\circ}\right)$ by up to $5 \mathrm{~dB}$. It is clear, however, that predictions of far off-normal angles of incidence for models using both positions of the appendages underestimate the actual backscattered TS by $5-10 \mathrm{~dB}$ or more. Furthermore, the scattering predictions show considerably more variability than the data associated with both tethered squid and freely swimming squid, although at least one set of measured data involved averages (tethered squid) that could contribute to the differences.

Additionally, comparisons were made between the phase-tracking DWBA predictions (high resolution morphometry, inhomogeneous case) and DWBA predictions us- ing a homogeneous prolate spheroid. When these models are compared at normal, dorsal incidence there are significant differences noticed in the structure of the frequency response [Fig. 8(a)]. While the prolate spheroid has a very regularly spaced interference pattern, the frequency response predicted by the phase-tracking DWBA method for the complex morphology of the squid tends to vary in both null spacing and peak amplitude.

\section{Effect of tilt angle on scattering predictions}

Scattering predictions versus tilt angle for dorsal aspect backscattering by $T$. pacificus are compared to predictions using the phase-tracking DWBA method (high resolution morphometry, inhomogeneous material properties, and straight- and splayed-arms cases) and DWBA predictions for a homogeneous prolate spheroid of equivalent volume and similar radius (Fig. 9). The frequency of the incident wave, as a model parameter, was chosen to correspond to a value of $k_{1} L_{\mathrm{ml}}$ that matched the frequency and mantle length of squid used in the published study of scattering by squid. The published results of live squid ensonified at $38 \mathrm{kHz}$ (Kang et al., 2005) include freely swimming squid, ensonified by a splitbeam transducer with swimming angle determined from perpendicular side-view cameras, and anesthetized squid tethered at various angles of tilt. The phase-tracking DWBA 


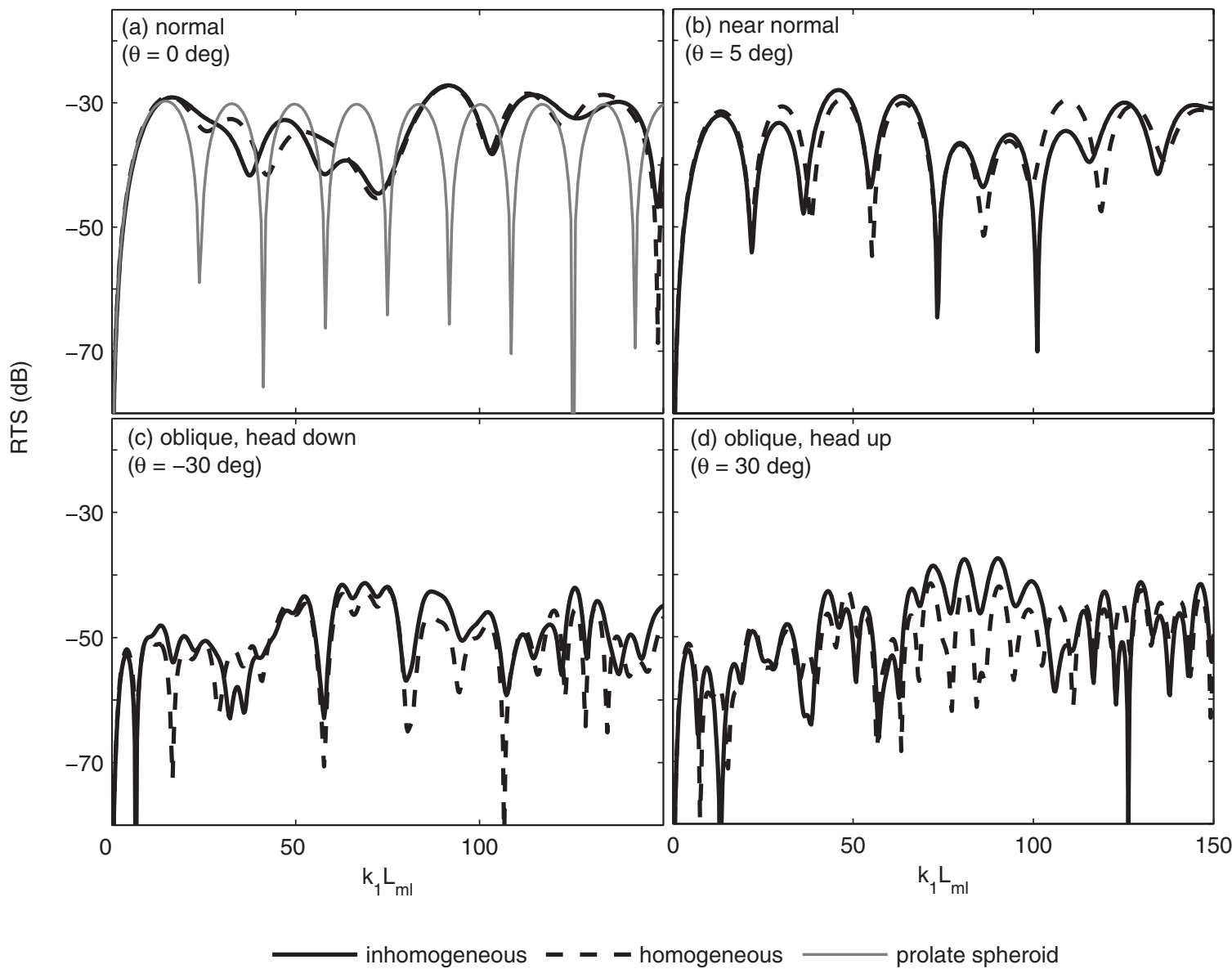

FIG. 8. Predictions of scattering by squid (T. pacificus) for various fixed orientations using scaled morphometry of L. pealeii (splayed arms) with homogeneous material properties (dashed lines) and inhomogeneous material properties, which includes interior structure of mantle cavity (solid lines). Predictions using the DWBA-based prolate spheroid model are shown for normal incidence only (solid gray line).

predictions (straight-arms case) agree well between $-20^{\circ}$ and $+30^{\circ}$ tilt in the anesthetized case. This model is significantly more accurate than the prolate spheroid model which strongly underpredicts the observed scattering levels at angles off-normal incidence $\left(|\theta| \geqslant 5^{\circ}\right)$.

\section{Comparison of model predictions with averaged target strength measurements}

Scattering predictions with respect to tilt angle are made for squid by compiling phase-tracking DWBA predictions for two degree angle increments from $-50^{\circ}$ to $+50^{\circ}$. Results were weighted using two different tilt distributions of freely swimming squid from published sources (Kang et al., 2004; Arnaya et al., 1989a). Published measurements from three studies on TSs of T. pacificus are compared with these predictions (Fig. 10). The model predictions are cut off at a value of $k_{1} L_{\mathrm{ml}}$ of 150 corresponding to a minimum resolution, $\lambda / l_{\mathrm{vs}}$, of 12.5 . The acoustic measurements come from three sources. Measurements from Arnaya et al. (1989b, 1989c) were estimated from mean volume backscattering strength. The data from Kang et al. (2005) were measured from individual squid using split-beam transducers at 38 and $120 \mathrm{kHz}$. Coincident tilt distribution of squid measured in these studies was not available. Thus, the predictions pre- sented here use both tilt distributions to compare with measured data.

Phase-tracking DWBA predictions and published data were compared using differential backscattering amplitude, where the mean absolute error is defined as $\hat{E}$ $=\left\langle\left|\sigma_{\mathrm{bs}}^{\text {data }}-\sigma_{\mathrm{bs}}^{\mathrm{pred}}\right|\right\rangle$ and the root mean squared error is defined as $E_{\mathrm{rms}}=\sqrt{\left\langle\left|\sigma_{\mathrm{bs}}^{\mathrm{data}}-\sigma_{\mathrm{bs}}^{\mathrm{pred}}\right|^{2}\right\rangle}$. The phase-tracking DWBA predictions (splayed-arms case) using a tilt distribution of $[-4,11.1]$ showed better agreement $\left(\hat{E}=1.23 \times 10^{-4}, E_{\mathrm{rms}}\right.$ $=1.52 \times 10^{-4}$ ) than the corresponding predictions using the tilt distribution of $[-17.7,12.7]\left(\hat{E}=2.26 \times 10^{-4}, E_{\mathrm{rms}}=2.46\right.$ $\times 10^{-4}$ ), as seen in Fig. 10(a). Furthermore, the model predictions using the straight-arms hybrid morphometry of squid and a tilt distribution of $[-4,11.1][$ Fig. 10(b)] agreed most closely with the data $\left(\hat{E}=9.69 \times 10^{-5}, \quad E_{\mathrm{rms}}=1.25\right.$ $\left.\times 10^{-4}\right)$.

Finally, the phase-tracking DWBA predictions are compared with DWBA predictions for a homogeneous prolate spheroid of an equivalent volume and similar radius. Both predictions use a tilt distribution of $[-4,11.1]$ due to the better fit with observed scattering data. By incorporating the high resolution digitizing of the outer shape only (i.e., using a homogeneous splayed-arms squid scaled to match the aspect ratio of the measured species), an improvement of 

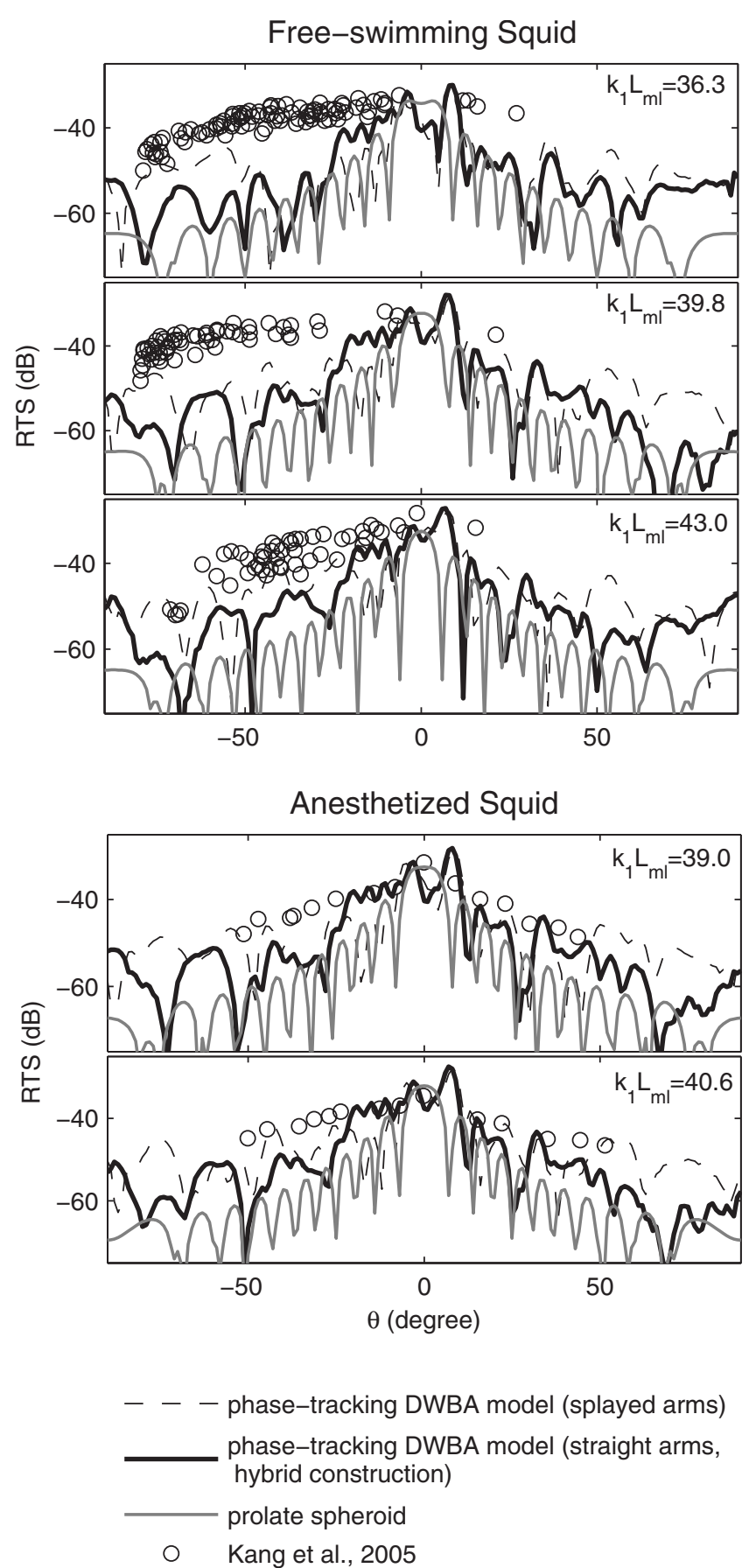

FIG. 9. Predictions of scattering by squid (T. pacificus) vs tilt angle using scaled morphometry of $L$. pealeii compared with experimental data. Black lines (solid and dashed) show phase-tracking DWBA predictions, with no averaging, using high resolution morphometry of $L$. pealeii and inhomogeneous material properties scaled to match the aspect ratio of $T$. pacificus. Dashed black lines show predictions using morphometry of the anesthetized squid (splayed-arms case). Solid black lines show predictions using hybrid morphometry of the two specimens of L. pealeii (straight-arms case). Solid gray lines show DWBA model predictions using an equivalent volume, homogeneous prolate spheroid with a similar radius. Predictions are compared with published measurements of $T$. pacificus (data points). Top three plots show data from live, freely swimming squid, ensonified at $38 \mathrm{kHz}$, while bottom two plots show data from anesthetized tethered squid also ensonified at $38 \mathrm{kHz}$ (Kang et al., 2005)

2-6 dB over the homogeneous prolate spheroid model was obtained for a wide range of $k a$ (results not shown). Replacing the homogeneous splayed-arms squid with an inhomogeneous straight-arms squid resulted in predictions with further improvements of generally $0-2 \mathrm{~dB}$. These improvements were through increases in level. In some narrow ranges of $k L$, the increases were 1-3 dB. Overall, the phase-tracking DWBA method, incorporating inhomogeneities and more realistic swimming positions (i.e., straight arms and tentacles), generally produced a 3-7 dB improvement in predictions over the homogeneous prolate spheroid model [Fig. 10(b)].

\section{DISCUSSION AND CONCLUSION}

A method that accurately accounts for scattering by an inhomogeneous weakly scattering object has been developed. This new method takes advantage of high resolution interior and exterior measurements of morphology and rigorously accounts for both the amplitude and phase of the scattered wave by using a phase-tracking approach to the DWBA. The inhomogeneous object can take the form of (1) variations of material properties within the body of the scatterer and/or (2) appendages or a part of the body that may be oriented such that the incident sound wave first passes through part of the body, into the surrounding fluid, and then back into another part of the body. This numerical method is applicable for all frequencies and all angles of scatterer orientation and can be applied to arbitrarily shaped threedimensional objects. The only constraint in the weakly scattering formulation is that the material properties (density and sound speed) may not deviate by more than several percent of the surrounding fluid.

The phase-tracking DWBA method has been tested against modal-series-based solutions to spherical and cylindrical shells and the DWBA finite-length cylinder model (closed form). There was excellent agreement in the comparisons over the entire range of shell thickness and tilt angle both with respect to overall scattering levels and structure of the scattering (i.e., position of the nulls). Furthermore, the model predictions agreed well with the analytic solutions to scattering over the range of material properties considered in this study. Additionally, an important result emerged from the modal-series-based solutions for scattering by objects of varying shell thicknesses. It has been shown here that the nonaveraged broadband frequency response of a weakly scattering inhomogeneous object cannot be accurately modeled by a homogeneous object of the same outer form.

The phase-tracking approach, in part, addresses a longstanding issue associated with predicting backscattering by weakly scattering, inhomogeneous, and/or irregularly shaped elongated bodies at angles well away from normal incidence. It has been shown in previous studies that predictions of scattering by elongated zooplankton, averaged over orientations near normal incidence, are relatively insensitive to small variations in shape and material properties (Stanton and $\mathrm{Chu}, 2000$ ). This has been attributed to the dominance of scattering from the front and back interfaces of the elongated scatterer. It has also been demonstrated that once the organism is oriented well-off broadside, these interfaces contribute less, making variations of shape and material property significant. This issue is common to a wide range of species of marine organisms, including commonly occurring zooplankton such as copepods and euphausiids. There have been at- 


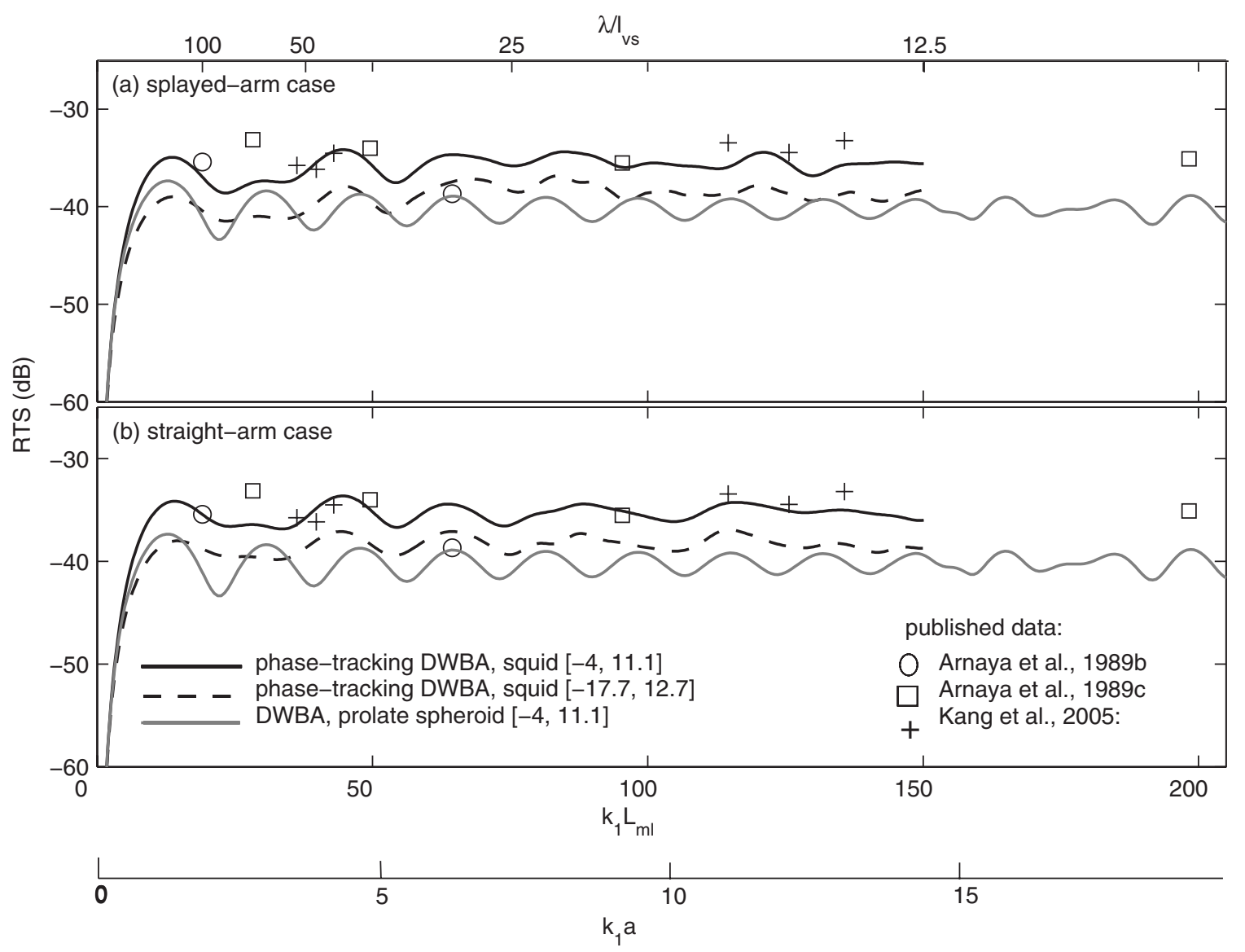

FIG. 10. Average scattering predictions for squid (T. pacificus) compared with experimental data. Predictions of scattering by squid use inhomogeneous material properties and (a) scaled morphometry of the anesthetized L. pealeii and (b) hybrid morphometry of the two specimens of $L$. pealeii to incorporate more natural swimming positions of the squid's arms and tentacles, also scaled. All predictions are averaged over the tilt angle using normal distributions limited to \pm 2 standard deviations of the mean. The two tilt-angle distributions presented use mean angle and standard deviation, $[\bar{\theta}, s]$, from published sources of swimming squid: $[-4,11.1]$ from Arnaya et al. (1989a), solid black lines, and [-17.7,12.7] from Kang et al. (2004), dashed black lines. Solid gray lines show DWBA model predictions using an equivalent volume, homogeneous prolate spheroid with a similar radius. Predictions are compared with published measurements of live, freely swimming squid, T. pacificus: circles (Arnaya et al., 1989b) squares (Arnaya et al., 1989c), and plus signs (Kang et al., 2005).

tempts to address this issue in earlier studies by modeling the shape of the organisms at high resolution (i.e., resolution cells much smaller than an acoustic wavelength). For example, in Stanton et al. (1998), the body of a euphausiid was assumed to be randomly rough at scales of $\lambda / 10$, with circular symmetry at each point of the body; in Stanton and Chu (2000), the outer boundaries of both euphausiids and copepods were digitized at $\lambda / 10$, again with circular symmetry at each point; in Lavery et al. (2002), CT scans were used to digitize the outer boundary in three dimensions at a minimum resolution of $\lambda / 10$; in Amakasu and Furusawa (2006) appendages were separately digitized and incorporated into the modeling of the scattering by euphausiids. Furthermore, some of these studies included, quite heuristically, inhomogeneities within the body by applying various profiles of material properties to the scattering object. In each of the studies, the backscattering levels for angles well-off-normal incidence were shown to increase significantly due to incorporation of the fine features of the body (surficial roughness, inhomogeneities within the body, appendages). However, none of the studies accounted for the fact that the phase change of the acoustic wave is altered as a result of passing through an inhomogeneity of differing sound speed. An important consequence of the phase-tracking approach is the inherent improvement in predictions of scattering at offnormal angles of incidence where inhomogeneities contribute significantly to the scattering.

The phase-tracking DWBA method also directly addresses the issues associated with modeling the scattering by squid. Laboratory data show that the scattering by squid is strongly dependent on tilt angle. Since free-swimming squid are known to swim at tilt angles roughly $4^{\circ}-20^{\circ}$ away from horizontal, quantitative interpretation of data from vertically aimed echosounders is one case requiring a scattering model that is accurate over a range of orientation angles. Prior to this study, the most advanced model of squid assumed it to be a homogeneous prolate spheroid that did not account for details of the shape of the body or inhomogeneities. As shown in our studies, the prolate spheroid model produces reasonable predictions (when compared with laboratory data) for the scattering by squid at near-normal dorsal incidence. However, our predictions also show that this model significantly underpredicts the scattering for angles away from normal incidence. When measured data and predictions are av- 
eraged over the observed distribution of swimming angles, the prolate spheroid again significantly underpredicts the data. By incorporating high resolution measurements of the outer shape and internal structure of squid obtained from spiral CT scans, the phase-tracking DWBA method produces significantly improved predictions over the DWBA, homogeneous prolate spheroid model. Improvements were seen in the TS levels for both single-orientation and tilt-averaged predictions and were most notable for single-orientation predictions at a range of off-normal angles of incidence.

Improvements in the scattering predictions for squid over earlier methods are a result of three aspects of the modeling of shape. These included high resolution depiction of the outer form of the squid body, internal inhomogeneities, and natural swimming position of squid appendages (e.g., arms and tentacles). When combined, these additional levels of complexity yielded a 3-7 dB improvement in the tiltaveraged predictions. Note that an improvement of $3 \mathrm{~dB}$ corresponds to a factor of 2 improvement in estimates of numerical density of the squid. As a result of these improvements, the tilt-averaged predictions with the new method are generally consistent with measured data over a wide range of $k a$. Also, for predictions at single angles of orientation, at or near-normal incidence, there were differences of 1-3 dB between the homogeneous and inhomogeneous cases.

Although broadband measurements of scattering by squid were not available for this study, another implication of rigorously accounting for inhomogeneities in a DWBA scattering model is that more accurate predictions may be made of frequency responses of weakly scattering marine organisms at discrete angles of orientation. Information about the frequency-dependent structure of individual echoes from organisms such as squid may aid in studies of prey discrimination by echolocating toothed whales and in the use of broadband scientific echo sounders for spectral classification of organisms.

For angles of incidence well away from normal incidence (and also well away from expected swimming angles), the new approach still significantly underpredicts the experimental data. There could be several sources of this discrepancy. For example, in this method it is assumed that the entire body is a weak scatterer where the material properties deviated from the surrounding fluid by only several percent. However, it is possible that there are small portions of the body that have much higher density and sound speed contrasts and can contribute significantly to the scattering under some conditions. These portions include a long thin gladius (or pen), a parrotlike beak, statoliths (small, bony inner ear organs), a hard cartilaginous cranium, and hard lenses in the eye (Williams, 1909). Iida et al. (2006) also identified the liver, a low density organ surrounded by higher density tissue, as a likely scatterer of sound. There are also uncertainties in the degree to which the tissue is inhomogeneous and there are uncertainties associated with the exact positioning of the appendages of the squid. Since the scattering by the main interfaces of the body is small at large angles of incidence, any of these other sources (or some combination) might contribute significantly to the scattering. These other sources are not a particular concern in this study since they do not appear to contribute significantly to the scattering at tilt angles associated with the swimming position of squid. However, in applications where the angles are much larger, those other sources need to be studied such as through the methods described in Nash et al. (1987) or Iida et al. (2006).

In conclusion, a new method for predicting acoustic scattering by weakly scattering bodies has been developed through applying a phase-tracking approach to the DWBA. This approach rigorously accounts for phase shifts due to inhomogeneities and appendages of the body. Furthermore, it makes use of high resolution three-dimensional digitizing of the body through spiral CT scans of the body. When applied to squid, the approach represents a significant improvement over the previous model of a low resolution homogeneous prolate spheroid. The improvements were based entirely on measured quantities and did not rely on any "floating" parameters. Although the application in this paper involved squid, this approach has the capability of making accurate predictions of scattering by a wide variety of important marine organisms in the fluidlike anatomical group, including euphausiids and copepods. The main challenges in all of the modeling will be the determination of material properties (sound speed and density) and orientation distribution, accounting for parts of the bodies that are not weak scatterers and obtaining sufficiently high resolution measurements of shape for small organisms acoustically sampled at high frequencies.

\section{ACKNOWLEDGMENTS}

The authors would like to thank Dr. Darlene Ketten at Woods Hole Oceanographic Institution and the colleagues in her group, particularly Julie Arruda, R.T.(R) who conducted the CT scans of squid presented here and Sr. Research Asst. Scott Cramer who aided in the handling, transport, and preservation of the specimens. The squid specimens used in this study were identified and provided by Ed Enos, Superintendent of the Aquatic Resources Division at Marine Biological Laboratory. We would also like to mention Dr. Roger Hanlon who provided great perspective concerning squid. Finally, the guidance and literature provided by Dr. Kohji Iida were most helpful in the comparisons with measured scattering by squid conducted in this research. Funding of this research was provided, in part, by the Office of the Oceanographer of the Navy and the Academic Programs Office at the Woods Hole Oceanographic Institution.

\section{APPENDIX: MODAL SERIES COEFFICIENTS FOR FLUID-FILLED FLUID SHELLS}

Modal series solutions for spherical and cylindrical scatterers have been derived for a wide range of material property profiles such as solid elastic, fluid, and fluid-filled elastic shells (Anderson, 1950; Goodman and Stern, 1962; Stanton, 1988; Faran, 1951). To the best of our knowledge, explicit expressions for the modal series coefficients for fluid-filled fluid shells have not been published; therefore, the following details are provided for spherical and cylindrical shells. In the following equations, the properties of the fluid surround- 
ing a scatterer are denoted by the subscript " 1 ," the fluid shell of the scatterer is denoted by subscript " 2 ," and the fluid inside the shell is denoted by the subscript " 3 " (Fig. 1).

\section{Spherical shells}

The $n$th modal series coefficient for a fluid-filled spherical fluid shell expressed as the variable, $A_{n}$, in Eq. (11) is given below as the ratio of the determinants of two matrices. These matrices contain the spherical Bessel functions of the first kind, $j_{n}$, the second kind, $\eta_{n}$, and the third kind (also known as the spherical Hankel functions of the first kind), $h_{n}^{(1)}$. Primes on the Bessel functions [e.g., $\left.h_{n}^{(1) \prime}\left(k_{1} a\right)\right]$ indicate derivatives with respect to their argument

$$
\begin{array}{|cccc}
A_{n}= & \left|\begin{array}{cccc}
j_{n}\left(k_{1} a\right) & 0 & -j_{n}\left(k_{2} a\right) & -\eta_{n}\left(k_{2} a\right) \\
j_{n}^{\prime}\left(k_{1} a\right) & 0 & -\frac{\rho_{1} c_{1}}{\rho_{2} c_{2}} j_{n}^{\prime}\left(k_{2} a\right) & -\frac{\rho_{1} c_{1}}{\rho_{2} c_{2}} \eta_{n}^{\prime}\left(k_{2} a\right) \\
0 & -j_{n}\left(k_{3} b\right) & j_{n}\left(k_{2} b\right) & \eta_{n}\left(k_{2} b\right) \\
0 & -\frac{\rho_{2} c_{2}}{\rho_{3} c_{3}} j_{n}^{\prime}\left(k_{3} b\right) & j_{n}^{\prime}\left(k_{2} b\right) & \eta_{n}^{\prime}\left(k_{2} b\right)
\end{array}\right| \\
\left|\begin{array}{cccc}
h_{n}^{(1)}\left(k_{1} a\right) & 0 & -j_{n}\left(k_{2} a\right) & -\eta_{n}\left(k_{2} a\right) \\
h_{n}^{(1) \prime}\left(k_{1} a\right) & 0 & -\frac{\rho_{1} c_{1}}{\rho_{2} c_{2}} j_{n}^{\prime}\left(k_{2} a\right) & -\frac{\rho_{1} c_{1}}{\rho_{2} c_{2}} \eta_{n}^{\prime}\left(k_{2} a\right) \\
0 & -j_{n}\left(k_{3} b\right) & j_{n}\left(k_{2} b\right) & \eta_{n}\left(k_{2} b\right) \\
0 & -\frac{\rho_{2} c_{2}}{\rho_{3} c_{3}} j_{n}^{\prime}\left(k_{3} b\right) & j_{n}^{\prime}\left(k_{2} b\right) & \eta_{n}^{\prime}\left(k_{2} b\right)
\end{array}\right|
\end{array}
$$

\section{Cylindrical shells}

The $n$th modal series coefficient for a fluid-filled cylindrical (infinite-length) fluid shell, expressed as the variable, $B_{n}$, in Eq. (12), is given below as the ratio of the determinants of two matrices. These matrices contain the cylindrical Bessel functions of the first kind, $J_{n}$, the second kind (also known as the Neumann functions), $N_{n}$, and the third kind (also known as the Hankel functions of the first kind), $H_{n}^{(1)}$. Primes on the Bessel functions [e.g. $\left.H_{n}^{(1) \prime}\left(k_{1} a\right)\right]$ indicate derivatives with respect to their argument,

$$
B_{n}=\frac{\left|\begin{array}{cccc}
J_{n}\left(k_{1} a\right) & 0 & -J_{n}\left(k_{2} a\right) & -N_{n}\left(k_{2} a\right) \\
J_{n}^{\prime}\left(k_{1} a\right) & 0 & -\frac{\rho_{1} c_{1}}{\rho_{2} c_{2}} J_{n}^{\prime}\left(k_{2} a\right) & -\frac{\rho_{1} c_{1}}{\rho_{2} c_{2}} N_{n}^{\prime}\left(k_{2} a\right) \\
0 & -J_{n}\left(k_{3} b\right) & J_{n}\left(k_{2} b\right) & N_{n}\left(k_{2} b\right) \\
0 & -\frac{\rho_{2} c_{2}}{\rho_{3} c_{3}} J_{n}^{\prime}\left(k_{3} b\right) & J_{n}^{\prime}\left(k_{2} b\right) & N_{n}^{\prime}\left(k_{2} b\right)
\end{array}\right|}{\left|\begin{array}{cccc}
H_{n}^{(1)}\left(k_{1} a\right) & 0 & -J_{n}\left(k_{2} a\right) & -N_{n}\left(k_{2} a\right) \\
H_{n}^{(1) \prime}\left(k_{1} a\right) & 0 & -\frac{\rho_{1} c_{1}}{\rho_{2} c_{2}} J_{n}^{\prime}\left(k_{2} a\right) & -\frac{\rho_{1} c_{1}}{\rho_{2} c_{2}} N_{n}^{\prime}\left(k_{2} a\right) \\
0 & -J_{n}\left(k_{3} b\right) & J_{n}\left(k_{2} b\right) & N_{n}\left(k_{2} b\right) \\
0 & -\frac{\rho_{2} c_{2}}{\rho_{3} c_{3}} J_{n}^{\prime}\left(k_{3} b\right) & J_{n}^{\prime}\left(k_{2} b\right) & N_{n}^{\prime}\left(k_{2} b\right)
\end{array}\right| .}
$$


Amakasu, K., and Furusawa, M. (2006). "The target strength of Antarctic krill (Euphausia superba) measured by the split-beam method in a small tank at $70 \mathrm{kHz}$," ICES J. Mar. Sci. 63, 36-45.

Anderson, V. C. (1950). "Sound scattering from a fluid sphere," J. Acoust. Soc. Am. 22, 426-431.

Arnaya, I. N., and Sano, N. (1990). "Studies on acoustic target strength of squid VI. Simulation of squid target strength by prolate spheroidal model," Bull. Fac. Fish., Hokkaido Univ. 41, 32-42.

Arnaya, I. N., Sano, N., and Iida, K. (1989a). "Studies on acoustic target strength of squid II. Effect of behaviour on averaged dorsal aspect target strength," Bull. Fac. Fish., Hokkaido Univ. 40, 83-99.

Arnaya, I. N., Sano, N., and Iida, K. (1989b). "Studies on acoustic target strength of squid III. Measurement of the mean target strength of small live squid," Bull. Fac. Fish., Hokkaido Univ. 40, 100-115.

Arnaya, I. N., Sano, N., and Iida, K. (1989c). "Studies on acoustic target strength of squid IV. Measurement of the mean target strength of relatively large-sized live squid," Bull. Fac. Fish., Hokkaido Univ. 40, 169-181.

Chu, D. Z., Foote, K. G., and Stanton, T. K. (1993). "Further analysis of target strength measurements of Antarctic krill at 38 and $120 \mathrm{kHz}$ : Comparison with deformed cylinder model and inference of orientation distribution," J. Acoust. Soc. Am. 93, 2985-2988.

Chu, D. Z., Wiebe, P., and Copley, N. (2000). "Inference of material properties of zooplankton from acoustic and resistivity measurements," ICES J. Mar. Sci. 57, 1128-1142.

Faran, J. (1951). "Sound scattering by solid cylinders and spheres," J. Acoust. Soc. Am. 23, 405-418.

Goodman, L. (1990). "Acoustic scattering from ocean microstructure," J. Geophys. Res. 95, 11557-11573.

Goodman, R. R., and Stern, R. (1962). "Reflection and transmission of sound by elastic spherical shells," J. Acoust. Soc. Am. 34, 338-344.

Hashimoto, T., and Maniwa, Y. (1952). "Studies on fish finder III. Studies on sound speed in fish body," Tech. Rep. Fish. Boat 3, 208-215.

Hofer, M. (2000). CT Teaching Manual (Thieme, New York).

Horne, J. K. (2000). "Acoustic approaches to remote species identification: A review," Fish. Oceanogr. 9, 356-371.

Iida, K., Takahashi, R., Tang, Y., Mukai, T., and Sato, M. (2006). "Observation of marine animals using underwater acoustic camera," Jpn. J. Appl. Phys., Part 1 45, 4875-4881.

Johnson, M., Madsen, P. T., Zimmer, W. M. X., de Soto, N. A., and Tyack, P. L. (2004). "Beaked whales echolocate on prey," Proc. R. Soc. London, Ser. B 271, S383-S386.

Johnson, R. (1977). "Sound scattering from a fluid sphere revisited," J. Acoust. Soc. Am. 61, 375-377.

Jones, B. A., Stanton, T. K., Lavery, A. C., Johnson, M. P., Madsen, P. T., and Tyack, P. L. (2008). "Classification of broadband echoes from prey of a foraging Blainville's beaked whale," J. Acoust. Soc. Am. 123, 1753 1762.

Kang, D., Iida, K., Mukai, T., and Hwang, D. (2004). "Acoustic target strength of Japanese common squid, Todarodes pacificus, and important parameters influencing its TS: Swimming angle and material properties" in OCEANS'04 MTS/IEEE/TECHNO-OCEAN'04, Kobe, pp. 364-369.

Kang, D., Iida, K., Mukai, T., and Kim, J. (2006). "Density and sound speed contrasts of the Japanese common squid Todarodes pacificus and their influence on acoustic target strength," Fish. Sci. 72, 728-736.
Kang, D., Mukai, T., Iida, K., Hwang, D. J., and Myoung, J. G. (2005). “The influence of tilt angle on the acoustic target strength of the Japanese common squid (Todarodes pacificus)," ICES J. Mar. Sci. 62, 779-789.

Lavery, A. C., Schmitt, R. W., and Stanton, T. K. (2003). "High-frequency acoustic scattering from turbulent oceanic microstructure: The importance of density fluctuations," J. Acoust. Soc. Am. 114, 2685-2697.

Lavery, A. C., Stanton, T. K., McGehee, D. E., and Chu, D. (2002). "Threedimensional modeling of acoustic backscattering from fluid-like zooplankton," J. Acoust. Soc. Am. 111, 1197-1210.

Lavery, A. C., Wiebe, P. H., Stanton, T. K., Gareth, L. L., Benfield, M. C., and Copley, N. (2007). "Determining dominant scatterers of sound in mixed zooplankton populations," J. Acoust. Soc. Am. 122, 3304-3326.

Lawson, G. L., Wiebe, P. H., Ashjian, C. J., Chu, D., and Stanton, T. K. (2006). "Improved parametrization of Antarctic krill target strength models," J. Acoust. Soc. Am. 119, 232-242.

Madsen, P. T., Johnson, M., de Soto, N., Zimmer, W. M. X., and Tyack, P. (2005). "Biosonar performance of foraging beaked whales (Mesoplodon densirostris)," J. Exp. Biol. 208, 181-194.

McGehee, D. E., O’Driscoll, R. L., and Traykovski, L. V. M. (1998). "Effects of orientation on acoustic scattering from Antarctic krill at $120 \mathrm{kHz}$," Deep-Sea Res., Part II 45, 1273-1294.

Morse, P. M., and Ingard, K. U. (1968). Theoretical Acoustics (McGrawHill, New York).

Mukai, T., Iida, K., Sakaguchi, K., and Abe, K. (2000). "Estimations of squid target strength using a small cage and theoretical scattering models," Proc. JSPS-DGHE Int. Symp. 10, 135-140.

Nash, D. M., Sun, Y., and Clay, C. S. (1987). "High resolution acoustic structure of fish,” J. Cons., Cons. Int. Explor. Mer 43, 23-31.

Roper, C., Sweeney, M., and Nauen, C. (1984). Cephalopods of the World. An Annotated and Illustrated Catalogue of Species of Interest to Fisheries, Vol. 3 (FAO, Rome).

Simmonds, E. J., and MacLennan, D. N. (2005). Fisheries Acoustics, Fish and Fisheries (Blackwell Science Ltd., Oxford).

Stanton, T. K. (1988). "Sound scattering by cylinders of finite length. I. Fluid cylinders," J. Acoust. Soc. Am. 83, 55-63.

Stanton, T. K., and Chu, D. (2000). "Review and recommendations for the modelling of acoustic scattering by fluid-like elongated zooplankton: Euphausiids and copepods," ICES J. Mar. Sci. 57, 793-807.

Stanton, T. K., Chu, D., and Wiebe, P. H. (1998). "Sound scattering by several zooplankton groups. II. Scattering models," J. Acoust. Soc. Am. 103, 236-253.

Stanton, T. K., Chu, D., Wiebe, P. H., and Clay, C. S. (1993). "Average echoes from randomly oriented random-length finite cylinders: Zooplankton models," J. Acoust. Soc. Am. 94, 3463-3472.

Starr, R., and Thorne, R. (1998). "Acoustic assessment of squid stocks," in Squid Recruitment Dynamics. The Genus Illex as a Model: The Commercial Illex Species. Influences on Variability, edited by E. O. R. Rodhouse and P. G. Dawe (FAO, Rome).

Tatarski, V. I. (1961). Wave Propagation in a Turbulent Medium (McGrawHill, New York).

Williams, L. W. (1909). The Anatomy of the Common Squid, Loligo pealii, Lesueur (Library and Printing Office, Leiden).

Yeh, C. (1967). "Scattering of acoustic waves by a penetrable prolate spheroid. I. Liquid prolate spheroid,” J. Acoust. Soc. Am. 42, 518-521. 